\title{
Improving 3D printing of garments by using HPC Cloud
}

\author{
D. Tomić*, D. Davidović*, T. Šubić**, J. Mesarić***, K. Skala* \\ * Ruđer Bošković Institute/ Center for Informatics and Computing, Zagreb, Croatia \\ ** Arctur d.o.o/Nova Gorica, Slovenia \\ ***Faculty of Electrical Engineering and Computing, Zagreb, Croatia
}

\begin{abstract}
D garment printing is a technology that is experiencing rapid development, and more and more garments are being printed on 3D printers. Advanced processes such as Selective Laser Sintering and PolyJet 3D printing are used. However, 3D printing of clothes still experiences some disadvantages, of which the biggest is the slow 3D printing speed and usually a small printing area of 3D printers. The novel, large multi-head printers can significantly increase the printing speed, but such printers require an additional step to prepare given $3 D$ models that increase. However, pre-processing time grows exponentially with the number of printer heads, therefore computer resources that exceed the capabilities of a single workstation are required to prepare a printout. This implies the use of HPC resources, and because of its flexibility, lower cost and ease of use, HPC Cloud is optimal platform for such jobs. We have shown that the structure of these jobs fits perfectly in the HPC Cloud environment.
\end{abstract}

Keywords - 3D garment printing, HPC Cloud, multi-head printers, PolyJet 3D printing, Selective Laser Sintering

\section{INTRODUCTION}

The connection between fashion [1] and 3D printing [2] is great and more and more projects are emerging. New additives [3] are coming, printing is becoming more precise, and new applications [4] are appearing. 3D printing offers huge opportunities for the fashion industry, from 3D printed clothing to 3D printed footwear and accessories, the possibilities are endless. Primarily, 3D printing was used more for an artistic touch, but the fashion industry is now moving towards the development of 3D printed wearable garments. The production of additives is interesting for fashion because it makes it easier to work on fashion designs and create amazing things for the fashion industry like clothes, jewelry and nets. This technology gives designers a lot of freedom in terms of geometry. For example, it is possible to create intricate designs for various projects within the fashion industry. From shoes and accessories to $3 \mathrm{D}$ printed dresses, the fashion industry is starting to embrace the full potential of $3 \mathrm{D}$ printing and develop interesting items.
With the current developments in 3D technology it becomes cheaper, more affordable and easier to use. The new opportunities in the fashion industry are arising and the reasons why designers are choosing $3 \mathrm{D}$ printing is changing: 3D printing turns out to be more sustainable and eco-friendlier. For instance, the production of textile generates the waste problem around the world, that is why a lot of elements of the manufacturing process need to be rethought in order to be eco-friendlier. Using 3D printing allows you to reduce waste, for example the $3 \mathrm{D}$ printer uses the exact amount of material that is needed to create a product. Experimenting with and creating new materials used in $3 \mathrm{D}$ printing in a more responsible way is the other reason for using $3 \mathrm{D}$ printing for fashion companies. The use of 3D printing to make clothes is constantly evolving. Extremely complex and impressive pieces were created first. The use of $3 \mathrm{D}$ printing in this sector is at a turn point to push the boundaries of the fashion world to create visually impressive creations. Nowadays, some designers are more interested in using the $3 \mathrm{D}$ printing technology to make their collections of regular clothing, using additive manufacturing, rather than using standards method of making. They use 3D printing not only for design benefits, but to create customizable and comfortable clothing. The introduction of advanced additives is a good way for all industries to improve their production process. It allows designers to work on their prototypes with faster and cheaper methods, and to produce customizable and cheaper products. 3D printing is a perfect technique for mass customization, which can otherwise be very expensive. Relatively fast tailoring is possible thanks to 3D printing. 3D printing could also fully create clothing that adapts to outdoor conditions. 3D printing thus transcends the boundaries of high fashion. There are already several examples on the market. The Spider dress from Anouk Wipprecht [5] has mechanical arms that extend and pull in response to external stimuli when people approach. The owner's breath helps to occupy the defensive position of the robotic arms. The dress is completely printed with Selective Laser Sintering (SLS) technology [6]. This technology prints the object parts additively by sintering tiny particles of polymer powder to bond them. The part is made layer by layer, according to the 3D model. During 3D printing, the SLS printer preheats the powder material just below its melting point, to allow the laser to melt faster. The roller applies a layer 
of polymer powder, then the laser sinters the powder according to the $3 \mathrm{D}$ file and the fabrication platform is lowered before applying a new layer of powder. The procedure is repeated until the desired part is created. Julia Daviy [7] uses 3D printing to create biodegradable fashion. Her collection includes 3D printed dresses. Clothes created with 3D printing are usually not easy to wear. But that's not the case with Julie Daviy's clothes. Another example is the American designer Travis Fitch [8]. He prints fashion pieces with colorful multiple materials. The material is flexible to respond to body movements. The use of colors in this project is quite unique. This technique offers new possibilities because multicolor 3D printing is not often used in fashion projects. The Ministry of Supply [9] has developed a 3D printed knitted blazer. The process of $3 \mathrm{D}$ printing and the work on the $3 \mathrm{D}$ design they create allows the piece to be adapted to the body and its movements. Their goal is to develop an agile supply chain and create products that are more comfortable and durable. Fashion designer Danit Peleg [10] decided to 3D print her entire fashion collection. It took about 2,000 hours to print it completely. Her goal is to create comfortable clothes that she could wear herself. Alexis Walsh [11] created an impressive 3D dress printed using Selective Laser Sintering. The dress has 400 tiles assembled by hand. It took the designer six months to develop this garment. Designer dresses are not always comfortable because that is not their main goal. However, by 3D scanning the model, Jessica Rosenkrantz [12] created a dress that perfectly fits her body. Another great example is Viptie 3D [13]. This company focuses more on the aspect of mass use of 3D printing. They create ties and strands for their customers. PolyJet 3D Printing [14] works similarly to inkjet printing, but instead of jetting drops of ink onto paper, PolyJet 3D Printers jet layers of curable liquid photopolymer onto a build tray. During the pre-processing, the software automatically calculates the placement of photopolymers and support material from a 3D CAD file. During the printing, the resin 3D printer jets and instantly UV-cures tiny droplets of liquid photopolymer. Fine layers accumulate on the build tray to create several highlydetailed 3D printed products. Where overhangs or complex shapes require support, the Polyjet 3D printer jets a removable support material, named FullCore 750 . The process of fine layer polymerization is repeated until the object is finished. After the photo polymerization is completed, the batch is placed under a pressurized water jet. This allows any excess liquid or supports to be removed with a few human interactions. The final product has a naturally smooth surface which can be polished further to create near full transparency for the clear resin. 3D printed models and parts are ready to handle and use right out after the cleaning process, there is no need for post-curing. There is a growth regarding the use of different 3D printing materials and techniques. In the future, metal 3D printing could be implemented to create some ornaments, but also laser cutting techniques.

\section{HPC CLOUD FOR 3D PRINTING}

Despite the great success of 3D printing in the garments production and design, one of the main drawbacks of this technology is the printing speed. As an example, Danit Peleg spent about 2,000 hours printing her collection, and Julia Daviy 6 months. This implies a higher price of such clothing, often unacceptable to the average customer. A multi-head printer with heads able to print a single object at the same time can significantly speed up printing [15], but then the time required to prepare the given 3D object (model) to the printer increases exponentially with the number of its heads. One solution to this problem is to leave the preparation of such files to the HPC system. However, traditional HPC resources are often difficult to access, and the cost of using them is relatively high, so it is good to consider using such resources on a Cloud system. In general, it is shown that a Cloud system is a suitable for HPC jobs in which no or minor inter-

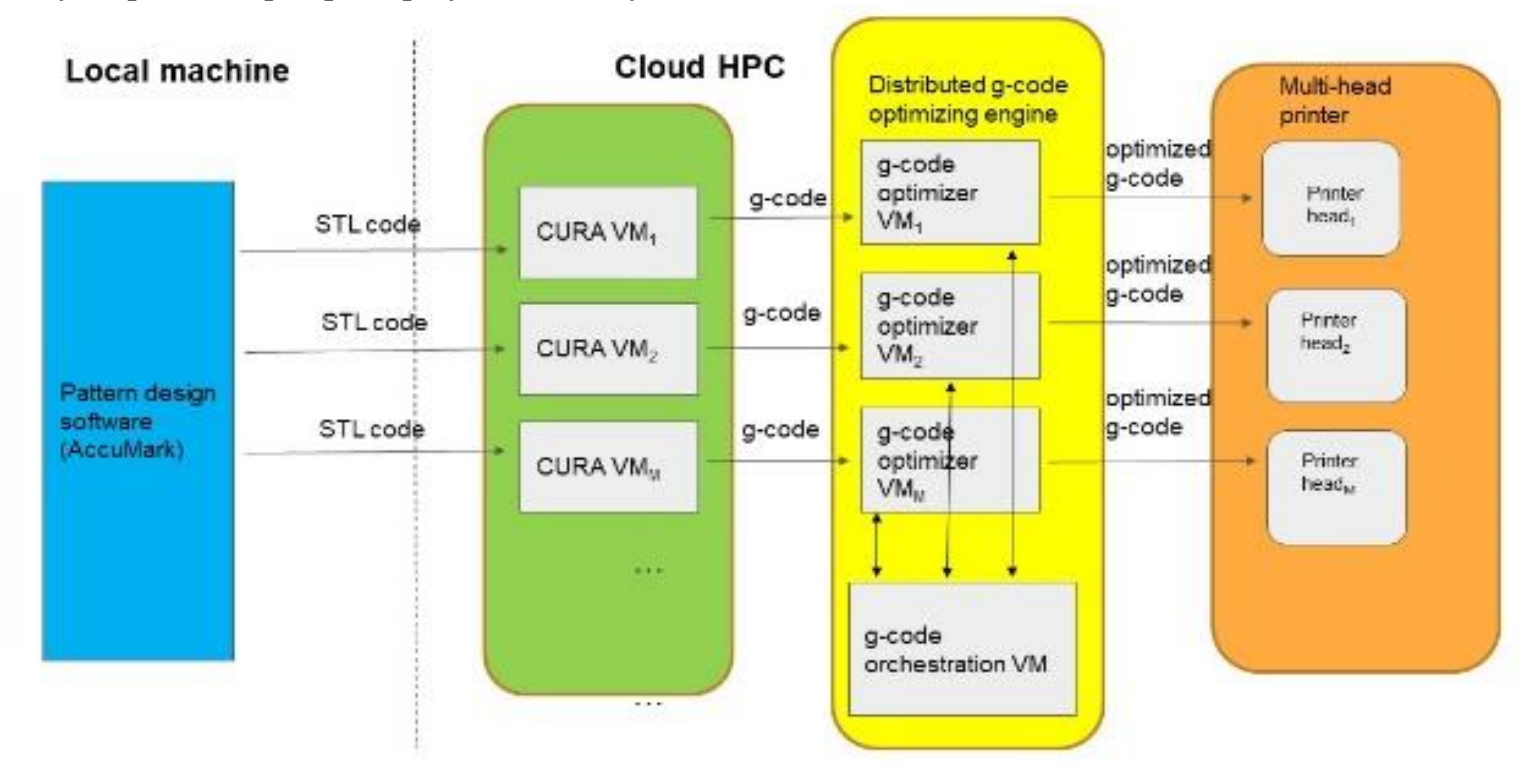

Fig 1: A schematic presentation of the 3D printing process in HPC Cloud. STL code fragments are generated on the local machine and sent to CURA virtual machines. These machines generate g-code and send it to the distributed g-code optimizing engine. Finally is generated and sent to the multi-headed printer. 
communication [16] is required. The calculation process is performed in two steps. In the first step, a code is generated from a CAD file (e.g. STL file) that describes the garments using a G-code generator, such as the CURA Ultimaker software [17]. G-code or RS274 is the most commonly used computer numerical control programming language, used to control automated machine tools, such as 3D printer. The G-code translates the 3D-generated model to a set of machine instructions. In the case of the standard, off-the-shelf 3D printers, no additional pre-processing is required. The obtained $\mathrm{G}$-codes are sent to the $3 \mathrm{D}$ printer(s) for printing. However, in the case of a specialized multi-head and/or multi-bridge 3D printers, the CURA generated G-code needs to be further optimized for that particular 3D printer. The whole process is schematically presented in Fig1. The goal of the optimization step is to divide the G-code, describing a single object that needs to be printed, into a set of G-codes, each tailored for one printing head. Then, each printing head can print its own part of the object without collision with other heads.

Let's the number of print heads be $\mathrm{M}$, and the number of layer $\mathrm{Ln}$ represented with matrix $A(i, j)$, with $N$ matrix elements of value 1 for points $i, j$ with print material. Then, the movement of $\mathrm{m}^{\text {th }}$ head between the certain points of value 1 can be represented as a function of $A$ :

$h m=f m(A) ; m=1, \ldots, M$

In addition, let each $\mathrm{m}^{\text {th }}$ head print $\mathrm{Nm}$ points. Then, the total distance $\mathrm{Hm}$ that $\mathrm{m}^{\text {th }}$ head needs to traverse is:

$H m=\sum_{i=1}^{N m} h m$

The total number of printed points by all heads is:

$N=N 1+N 2+\cdots+N m$

As the load should be equally distributed across all head, the following optimization problem can be posed:

$\operatorname{minimize}(\mathrm{Hm})$ w.r.t. $N 1=N 2=\cdots=N m$

Apparently, (4) can be split to $M$ independent parallel tasks, with the communication occurring between them when they need to interchange the optimization results. Minimizing $\mathrm{Hm}$ is similar to the well-known travelling salesman problem [18] and the number of computational steps (NCS) required is exponential to the number of points traversed:

$N C S=K m \exp ^{N m}$

Value $\mathrm{Km}$ is dependent of the shape of the printing segment $\mathrm{m}$. On the other side, the number of communications (NC) between tasks is:

$\mathrm{NC}=\mathrm{M} !$

The maximum number of printing heads $M$ within one 3D printer on today's market is 6 . Therefore, even for the objects with many segments the communication is neglectable to the processing:

$\mathrm{NCS} \ll$ NC

Inequality (7) suggests that the optimization of G-Code can be effectively performed on Cloud.

\section{CONCLUSION}

3D printing of clothing is increasingly coming to the fore today. However, this is a slow process, which increases the cost of its production. Multi-headed printers are able to print faster, but the pre-processing time of the required files grows proportionally with the number of printer heads. Fortunately, inequality (7) and the results from [16] suggests that this time can be significantly shortened by parallel processing these files on high-performance computing (HPC) systems. Further analysis of this problem shows that due to its specific structure, it can be efficiently solved on the Cloud. This increases the flexibility of the solution and reduces production costs.

\section{ACKNOWLEDGMENT}

This research has been supported by the European Commission H2020 project CloudiFacturing under grant agreement no. 768892 and by the European Regional Development Fund under the grant KK.01.1.1.01.0009 DATACROSS.

\section{REFERENCES}

[1] Laver J: The Concise History of Costume and Fashion, H. N. Abrams, New York, 1979.

[2] Ngo TD, Kashani A, Imbalzano G, Nguyen TK, Hui D. Additive manufacturing (3D printing): A review of materials, methods, applications and challenges. Composites Part B: Engineering, Volume 143, 15 June 2018, Pages 172-196

[3] Vaezi M, Chianrabutra S, Mellor B, Yang S. Multiple material additive manufacturing - Part 1: a review, Virtual and Physical Prototyping, 8:1, 19-50, 2013. DOI: $\underline{10.1080 / 17452759.2013 .778175}$

[4] Shahrubudin N, Lee T.C., Ramlan R. An Overview on 3D Printing Technology: Technological, Materials, and Applications. Procedia Manufacturing, Volume 35, 2019, Pages 1286-1296. https://doi.org/10.1016/j.promfg.2019.06.089

[5] https://www.arch2o.com/the-spider-dress-anouk-wipprecht/ [6] Kruth J.P., Mercelis P, Vaerenbergh V, Froyen L, Rombouts $\mathrm{M}$, Binding mechanisms in selective laser sintering and selective laser melting, Rapid Prototyping Journal, vol 11, issue 1, Feb. 2015.

[7] https://www.3dnatives.com/en/julia-daviy-280520194/

[8] https://www.futurecurrent.net/travis-fitch

[9] https://www.ministryofsupply.com/

[10] https://en.wikipedia.org/wiki/Danit_Peleg

[11] https://www.interlaced.co/article/keeping-up-with-alexiswalsh-1529511915

[12] https://n-e-r-v-o-u-s.com/about_us.php

[13] https://www.viptie3d.com/ 
[14] Meisel N.A., Elliott A.M., Williams C.B. A procedure for creating actuatedjoints via embedding shape memoryalloys in PolyJet 3D printing. Journal of Intelligent Material Systemsand Structures2015, Vol. 26(12) 1498-1512

[15] http://www.goya3d.com/\#xl_xr_page_index

[16] D. Tomić, Z. Car and D. Ogrizović, "Running HPC applications on many million cores Cloud," 2017 40th International Convention on
Information and Communication Technology, Electronics and Microelectronics (MIPRO), Opatija, 2017, pp. 209-214, doi: 10.23919/MIPRO.2017.7973420.

[17] https://ultimaker.com/software/ultimaker-cura

[18] Lawler E. L. (1985). The Travelling Salesman Problem: A Guided Tour of Combinatorial Optimization (Repr. with corrections. ed.). John Wiley \& sons. ISBN 978-0471904137. 